\title{
Nanofitas de óxido de estanho: controle do estado de oxidação pela atmosfera de síntese
}

\section{(Tin oxide nanobelts: oxidation state control by the synthesis atmosphere)}

\author{
M. O. Orlandi, R. Aguiar, M. R. D. Bomio, E. R. Leite, E. Longo \\ ${ }^{l}$ CMDMC-LIEC, Departamento de Química, Universidade Federal de S. Carlos \\ Rod. Washington Luiz km 235, C.P. 676, S. Carlos, SP, 13565-905 \\ porlandi@iris.ufscar.br
}

\begin{abstract}
Resumo
Nanofitas de óxido de estanho com diferentes estados de oxidação foram produzidas pelo método de redução carbotérmica a $1260{ }^{\circ} \mathrm{C}$. As nanoestruturas foram caracterizadas por difração de raios X, microscopia eletrônica de varredura e espectroscopia por dispersão de energia de raios X. O controle do estado de oxidação das nanoestruturas foi feito pela variação da atmosfera de síntese dentro do forno. Em atmosferas sem oxigênio os materiais obtidos foram nanofitas de $\mathrm{SnO}$ e de $\mathrm{SnO}_{2}$, enquanto em atmosferas com oxigênio (aproximadamente 10\%) obteve-se apenas o crescimento de nanofitas de $\mathrm{SnO}_{2}$.

Palavras-chave: nanofitas, óxido de estanho, atmosfera de síntese.
\end{abstract}

\begin{abstract}
Tin oxide nanobelts in different states of oxidation were produced by carbothermal reduction at $1260{ }^{\circ} \mathrm{C}$. The nanostructures were characterized by X-ray diffraction, scanning electron microscopy and energy dispersive X-ray spectroscopy. The state of oxidation of the nanostructures was controlled by the synthesis atmosphere inside the furnace. The materials obtained in atmospheres without oxygen were $\mathrm{SnO}$ and $\mathrm{SnO}_{2}$ nanobelts, while atmospheres with oxygen (about 10\%) only allowed for the growth of $\mathrm{SnO}{ }_{2}$ nanobelts.

Keywords: nanobelts, tin oxide, synthesis atmosphere.
\end{abstract}

\section{INTRODUÇÃO}

Nanotubos de carbono foram descobertos em 1991 por Iijima [1], que relatou que esses podem ser de dois tipos: os nanotubos de parede simples (NTPS) e os nanotubos de parede múltipla (NTPM). Os nanotubos de parede simples são formados a partir de uma folha de grafite enrolada com os extremos fechados por metades de fulerenos, e os nanotubos de parede múltipla são nanotubos de parede simples enrolados de tal forma que fiquem concêntricos.

Estimulados pelas novas propriedades obtidas em nanotubos de carbono, houve um grande esforço para sintetizar nanoestruturas a partir de materiais inorgânicos. Nanotubos de óxido de zinco foram sintetizados por Wang e $\mathrm{Li}$ [2] dentro dos poros de uma membrana nanoporosa de alumina [3, 4]. O estudo mostra que nanotubos policristalinos são obtidos para curtos tempos de imersão da membrana em uma solução à base de $\mathrm{ZnO}$, e que, para longos tempos de imersão, estruturas tipo fio (nanofios) são produzidas.

Recentemente, um novo tipo de nanoestrutura com morfologia tipo fita (nanofita), que possui seção transversal retangular, foi preparado a partir da evaporação térmica de óxidos metálicos [5]. Nanofitas de $\mathrm{ZnO}$ monocristalinas foram preparadas em fornos tipo tubo sem a presença de partículas catalisadoras, pelo método de evaporação térmica. Neste processo, óxido de zinco é colocado no centro de um forno tubo em que a temperatura, a pressão e o tempo de evaporação são controlados. Nanofitas de $\mathrm{In}_{2} \mathrm{O}_{3}, \mathrm{CdO}, \mathrm{Ga}_{2} \mathrm{O}_{3}, \mathrm{PbO}_{2}$ também foram produzidas pelo mesmo método e, de forma geral, mostraram-se monocristalinas.

Dióxido de estanho é um semicondutor do tipo-n [6], apresentando uma estrutura cristalina tetragonal do tipo rutilo e é bem conhecido para aplicações como sensor de gás [7], eletrodos condutores transparentes [8] e varistores [9]. Nos últimos anos, têm sido reportado que o $\mathrm{SnO}_{2}$ nanoestruturado possui propriedades equivalentes à do material na forma de pastilhas. Nanoestruturas de $\mathrm{SnO}_{2}$ foram primeiramente produzidas em 2001 [5]. Essas nanoestruturas possuíam secção transversal retangular (nanofitas) e eram monocristalinas [10].

Outros tipos de nanoestruturas, como nanofios e nanotubos de $\mathrm{SnO}_{2}$ também foram sintetizadas [11]. Os nanofios são de modo geral monocristalinos, embora contornos gêmeos tenham sido observados em algumas dessas nanoestruturas. Em outros nanofios, além da estrutura rutilo, foram encontradas algumas reflexões da estrutura ortorrômbica do $\mathrm{SnO}_{2}$. Os estudos mostram que essa estrutura só é obtida em pastilhas de $\mathrm{SnO}_{2}$ quando formadas sob condições de alta pressão [12].

Recentemente, um novo tipo de síntese (redução carbotérmica) foi proposto para a obtenção de nanofitas de $\mathrm{SnO}_{2}$ [13]. O material de partida utilizado foi o próprio $\mathrm{SnO}_{2}$ com 5\% em peso de "negro de fumo". Foi reportado [13] que o $\mathrm{SnO}_{2}$ pode ser reduzido para $\mathrm{SnO}$ ou mesmo $\mathrm{Sn}^{0}$ antes de passar pelo processo de evaporação. Isso faz com que a 
temperatura de obtenção das nanoestruturas seja sensivelmente diminuída sem que a morfologia das nanofitas obtidas seja diferente daquelas obtidas por outros métodos.

O objetivo deste trabalho foi produzir nanoestruturas de óxido de estanho com diferentes estados de oxidação, utilizando o método de redução carbotérmica [13], por meio do controle da atmosfera dentro do forno.

\section{PROCEDIMENTO EXPERIMENTAL}

As nanoestruturas foram preparadas pelo método de redução carbotérmica, que consiste basicamente na redução pelo carbono do óxido do qual se deseja produzir as nanoestruturas, seguido da evaporação do material resultante e da sublimação do vapor do óxido.

Na preparação do pó utilizado para a obtenção das nanoestruturas, misturou-se o $\mathrm{SnO}_{2}$ com $5 \%$ em peso de "negro de fumo". Após a mistura, o pó foi macerado em almofariz de ágata e forçado a passar em uma peneira de malha 100 mesh (abertura de $\approx 140 \mu \mathrm{m}$ ). Após a mistura entre o $\mathrm{SnO}_{2}$ e o "negro de fumo", o pó resultante foi colocado em navícula de alumina, que foi introduzida no centro de um forno tubular. As extremidades do tubo foram então fechadas com peças de teflon especialmente fabricadas, na qual há um orifício para a entrada (ou saída) do gás de arraste.

As queimas foram feitas na temperatura de $1260{ }^{\circ} \mathrm{C}$, com uma taxa de aquecimento e resfriamento de $15^{\circ} \mathrm{C} / \mathrm{min}$, e tempo de patamar de $2 \mathrm{~h}$. Os gases utilizados durante o processo de fabricação das fitas foram oxigênio, nitrogênio e uma mistura de nitrogênio e ar sintético (1/1). A vazão utilizada durante as queimas foi de $100 \mathrm{~cm}^{3} / \mathrm{min}$. O gás utilizado, além de propiciar a atmosfera desejada, também serve para arrastar o óxido na forma de vapor da região quente (centro) do forno e fazê-lo condensar em uma região mais fria, região em que as nanoestruturas são formadas.

As nanoestruturas foram caracterizadas por difração de raios X(Siemens, modelo D-5000), microscopia eletrônica de varredura (MEV) e por espectroscopia por dispersão de energia de raios X (EDX) utilizando um estágio acoplado ao microscópio eletrônico de varredura (Zeiss - DSM 940A). Para a preparação das amostras para MEV foi necessário fazer uma dispersão das nanoestruturas. Para isso, fez-se a imersão das nanoestruturas em etanol e então a mistura foi levada a um limpador ultra-sônico. Após a dispersão, uma parte da mistura foi colocada sobre um substrato de vidro que foi seco em estufa $\left(60^{\circ} \mathrm{C}\right)$ e recoberto com uma fina camada de ouro para proporcionar condutividade elétrica.

\section{RESULTADOS E DISCUSSÃO}

Após a calcinação, as amostras retiradas do forno possuíam diferentes colorações, dependendo do tipo de gás de arraste (atmosfera) utilizado (Figs. 1a e 1b). Quando o gás de arraste utilizado foi o oxigênio, nenhuma nanoestrutura foi obtida, e restou na navícula de alumina apenas um pó de cor clara. A análise por difração de raios $\mathrm{X}$ mostrou que esse pó possuía apenas a fase cassiterita do $\mathrm{SnO}_{2}$. O excesso de oxigênio na atmosfera oxida o carbono antes que o dióxido de estanho seja reduzido para $\mathrm{SnO}$; assim sendo, o pó resultante continua

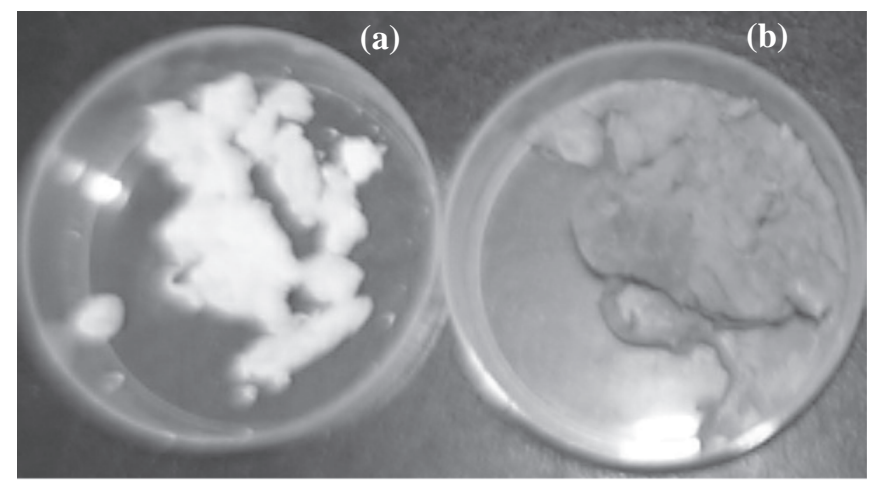

Figura 1: Foto do material como retirado do forno, mostrando a diferença das cores dependendo da atmosfera de queima: a) material produzido com atmosfera de nitrogênio e oxigênio em proporções iguais, b) material produzido com atmosfera de nitrogênio.

[Figure 1: Photo of the materials as removed from the furnace, showing the difference in color according to calcination at different atmospheres: a) material produced with a 1:1 ratio of synthetic air and nitrogen; b) material produced under nitrogen.]

sendo $\mathrm{SnO}_{2}$ e, portanto, não evapora na temperatura de $1260{ }^{\circ} \mathrm{C}$. Nanoestruturas partindo de $\mathrm{SnO}_{2}$ puro são obtidas apenas em temperaturas acima de $1350{ }^{\circ} \mathrm{C}$ [5].

Quando o gás de arraste utilizado foi nitrogênio, um material tipo "algodão" e de coloração escura (cinza) foi retirado das paredes do forno (Fig. 1b). Para a mistura em proporções iguais dos gases nitrogênio e ar sintético, um material também com a consistência de "algodão" foi retirado das paredes do forno; a diferença é que esse material possuía coloração branca (Fig. 1a). Esses materiais foram retirados do forno em uma região na qual a temperatura é entre 100 e $300^{\circ} \mathrm{C}$ menor que a região central do forno (região de evaporação).

A Fig. 2 mostra os difratogramas de raios $\mathrm{X}$ dos materiais em

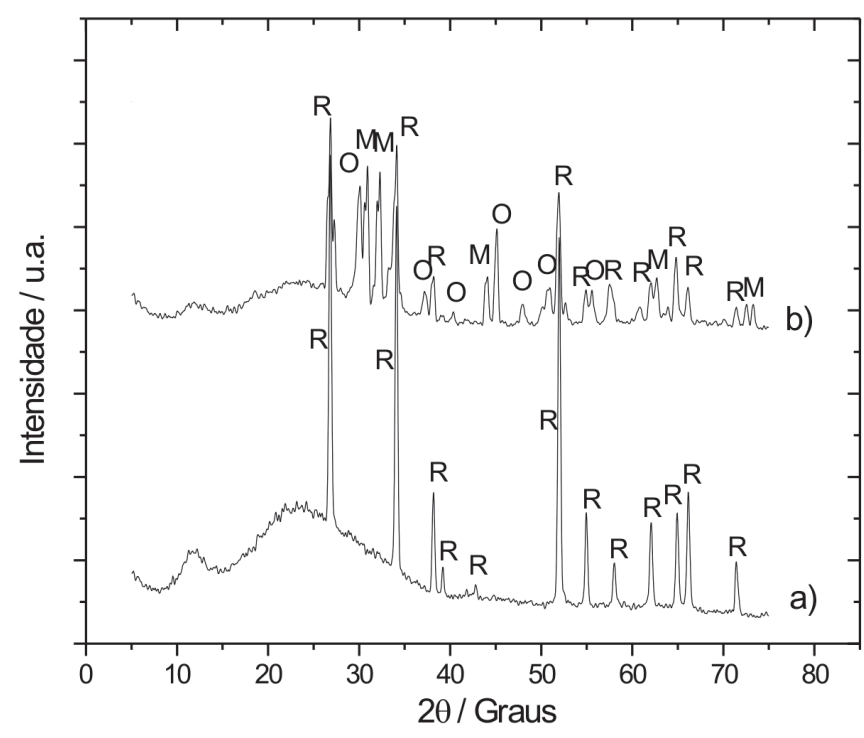

Figura 2: Difratogramas de raios $\mathrm{X}$ dos materiais sintetizados em diferentes atmosferas: a) mesma razão de ar sintético e nitrogênio e b) nitrogênio. [Figure 2: X-ray diffraction patterns of the materials calcined under different atmospheres: a) 1:1 ratio of synthetic air and nitrogen; $b$ ) nitrogen.] 
estudo. Observa-se que o material de cor branca é composto apenas por nanoestruturas com a estrutura rutilo (R) do $\mathrm{SnO}_{2}$ (Fig. 2a). Dentro do forno, devido a presença de nitrogênio e oxigênio, torna-se possível a oxidação das nanofitas formadas, portanto elas condensam no estado termicamente mais estável do óxido de estanho, que é o $\mathrm{SnO}_{2}$ [14]. O material de cor cinza apresentou, além da fase cassiterita do $\mathrm{SnO}_{2}$, a fase ortorrômbica $(\mathrm{O})$ do $\mathrm{SnO}$ e a fase do estanho metálico (M). Esse resultado mostra que é possível obter diferentes estados de oxidação de nanoestruturas de estanho, apenas controlando a atmosfera de síntese. As equações A-C ilustram as reações que devem estar ocorrendo para que esses diferentes estados de oxidação sejam obtidos.

Formação de $\mathrm{SnO}$ :

$$
2 \mathrm{SnO}_{2}+\mathrm{C} \longrightarrow 2 \mathrm{SnO}+\mathrm{CO}_{2(\mathrm{~g})}
$$

Formação do estanho metálico:

$$
2 \mathrm{SnO}+\mathrm{C} \longrightarrow 2 \mathrm{Sn}^{\circ}+\mathrm{CO}_{2(\mathrm{~g})}
$$

Formação do $\mathrm{SnO}_{2}$ :

$$
\mathrm{SnO}+1 / 2 \mathrm{O}_{2 \text { (ar sint.) }} \longrightarrow \mathrm{SnO}_{2}
$$

A Fig. 3 mostra fotomicrografias obtidas por microscopia eletrônica de varredura para as nanoestruturas de $\mathrm{SnO}_{2}$ (coloração branca). Observa-se pela Fig. 3 que essas nanoestruturas possuem a forma de fitas (nanofitas) e tamanhos que variam desde alguns nanômetros até centenas de micra. Algumas dessas nanoestruturas apresentam a forma de dendritas (Fig. 3b), ou seja, uma "nanofita primária" serve de base para o crescimento de outras nanofitas. Esse tipo de morfologia (nanodendritas) já foi reportado para nanoestruturas de $\mathrm{SnO}_{2}$
[15], mas seu mecanismo de formação ainda não está claro.

As nanoestruturas que possuem a cor cinza (Fig. 4) têm uma micrografia um pouco mais complexa. As nanoestruturas também possuem a forma de fitas, mas há em alguns pontos "esferas", a partir das quais parecem crescer as fitas. Uma análise feita por EDX (Fig. 5) mostrou que essas "esferas" são de estanho metálico, ou seja, não possuem oxigênio, o que está de acordo com o resultado de difração de raios $\mathrm{X}$, que apresentou os picos da fase estanho metálico. A falta de oxigênio na atmosfera do forno não possibilita as nanofitas voltarem ao seu estado mais oxidado $\left(\mathrm{SnO}_{2}\right)$ e, portanto, "esferas" de estanho metálico e fitas de $\mathrm{SnO}$ são preferencialmente formados.

Algumas nanofitas de $\mathrm{SnO}$ (cinza escura) foram calcinadas novamente a $1000{ }^{\circ} \mathrm{C}$ por $1 \mathrm{~h}$ na presença de oxigênio, e quando retiradas do forno apresentavam uma coloração branca. A análise do difratograma de raios $\mathrm{X}$ dessa amostra após a calcinação apresentou somente a fase cassiterita do $\mathrm{SnO}_{2}$, provando que o material retornou ao seu estado mais oxidado. As micrografias obtidas para a amostra após a calcinação não apresentaram diferença morfológica em relação à amostra antes da calcinação.

Devido as diferentes propriedades (elétricas, ópticas, etc.) que o estanho apresenta quando em diferentes estados de oxidação, o controle da formação desses estados de oxidação em nanoestruturas simplesmente pela atmosfera de queima é um avanço para futuras aplicações na área de nanotecnologia.

\section{CONCLUSÕES}

O controle da atmosfera do forno proporcionou a obtenção de nanoestruturas de estanho com diferentes estados de oxidação. Os resultados mostram que as nanoestruturas possuem secção transversal retangular (nanofitas) e que nanofitas de $\mathrm{SnO}_{2}$ e $\mathrm{SnO}$ foram obtidas. Para as nanofitas de $\mathrm{SnO}$, "esferas" de estanho metálico parecem servir de catalizadores para o

b)

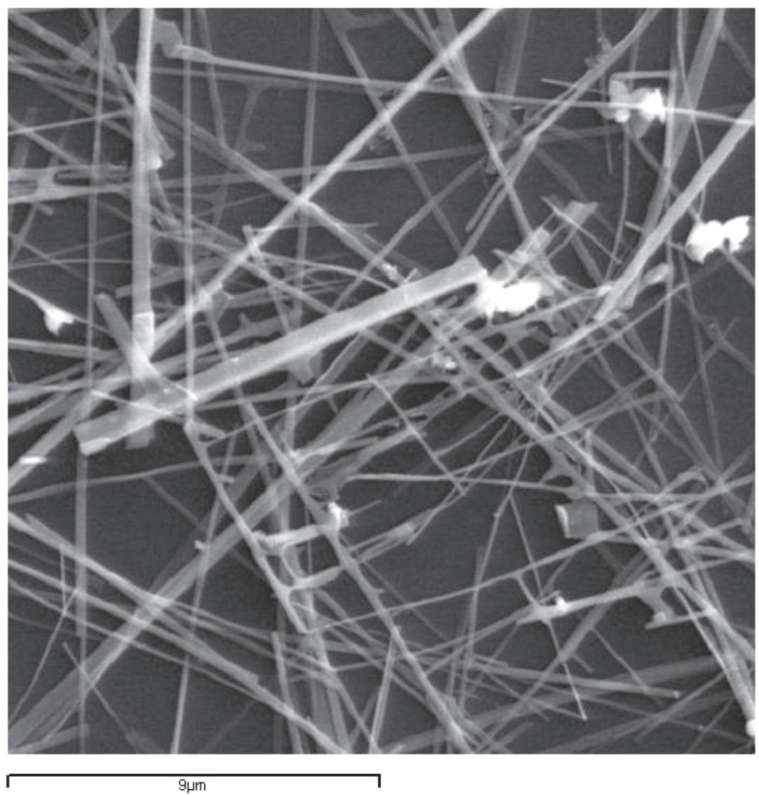

a)

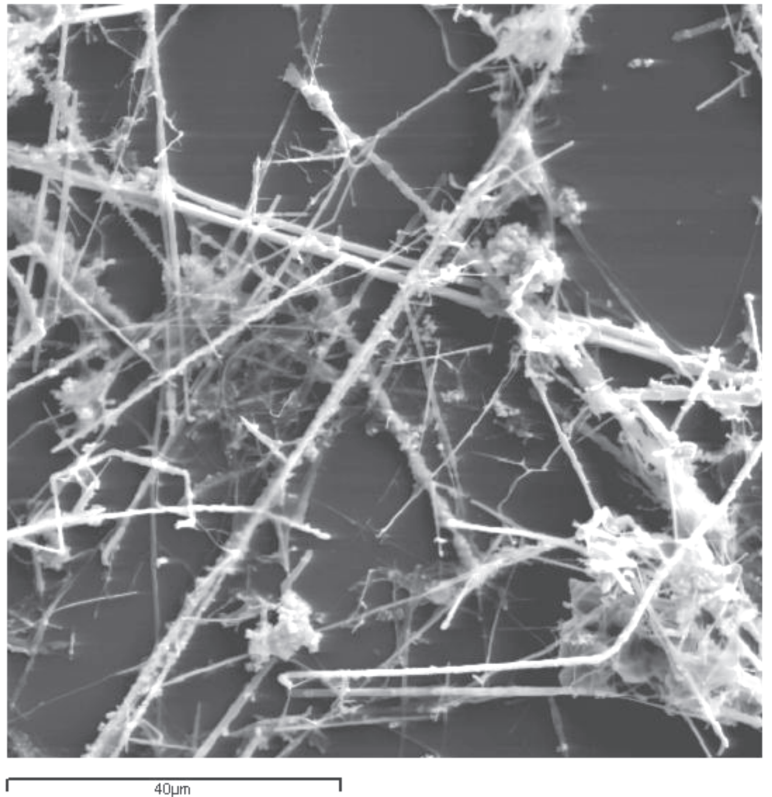



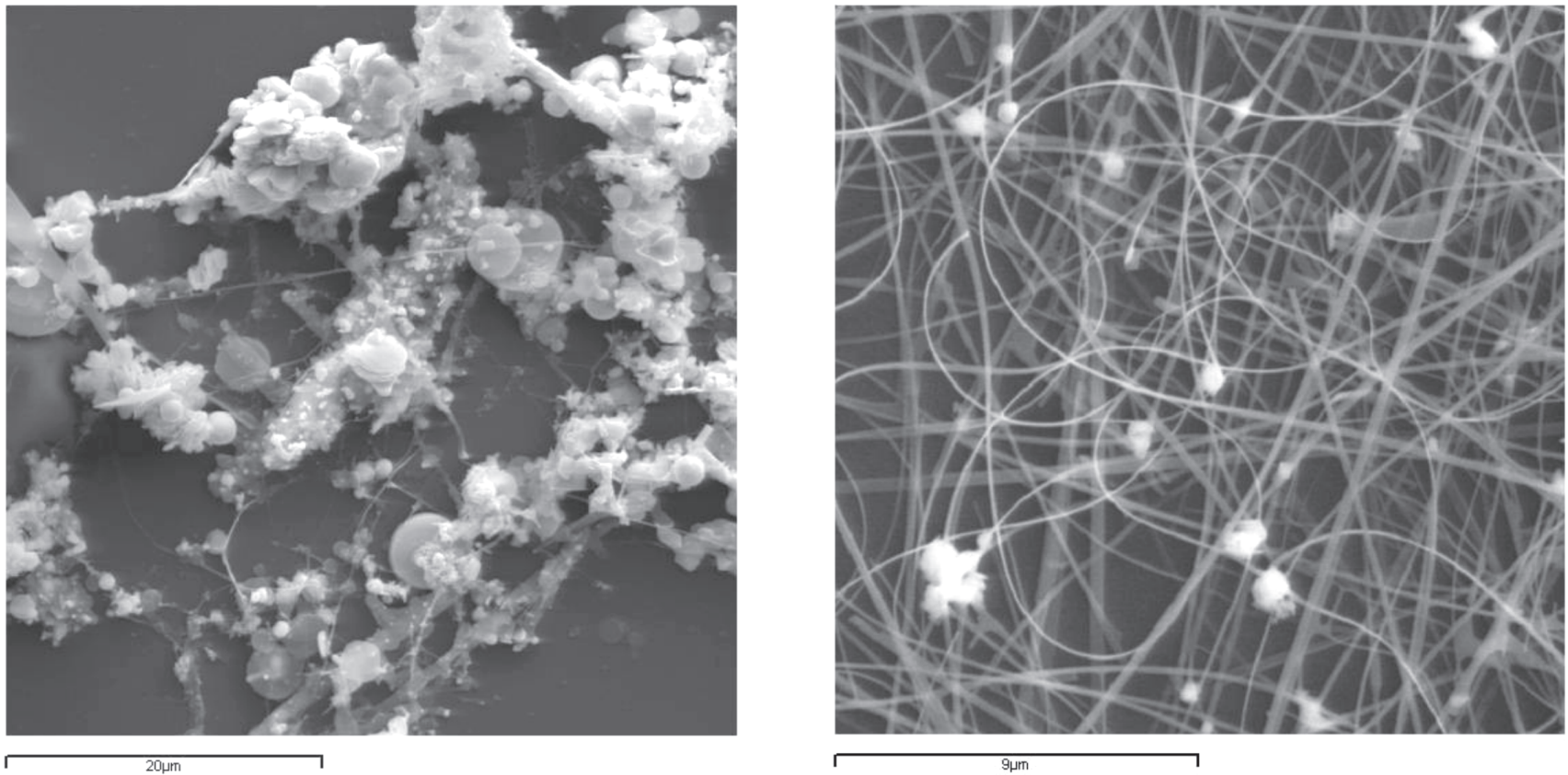

Figura 4: Micrografias obtidas por microscopia eletrônica de varredura da amostra de coloração cinza. [Figure 4: SEM micrographs of the dark (gray) sample.]
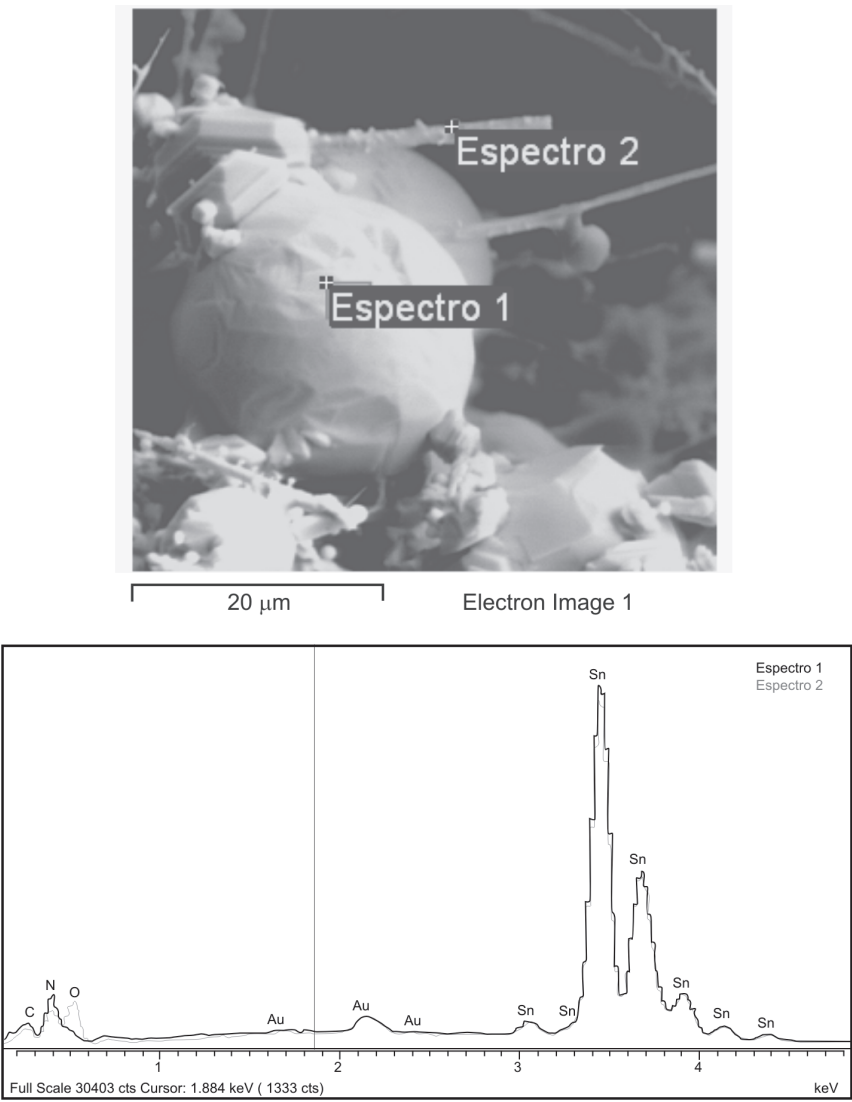

Figura 5: EDX da amostra de coloração cinza mostrando que as "esferas" não possuem oxigênio.

[Figure 5: EDX of the dark sample showing spheres devoid of oxygen.] crescimento das nanoestruras, pois geralmente são encontradas "esferas" em uma das extremidades das nanoestruturas.

\section{AGRADECIMENTOS}

Os autores agradecem ao CNPq, CEPID e FAPESP pelo auxílio financeiro na realização deste trabalho.

\section{REFERÊNCIAS}

[1] S. Iijima, Nature 354, 7 (1991) 56.

[2] Z. Wang, H. L. Li, Appl. Phys. A 74, 2 (2002) 201.

[3] C. R. Martin, Science 266 (1994) 1961.

[4] C. R. Martin, Chem. Mater. 8, 8 (1996) 1739.

[5] Z. W. Pan, Z. R. Dai, Z. L. Wang, Science 291, 9 (2001) 1947.

[6] Z. M. Jarzebski, J. P. Marton, J. Eletrochem. Soc. 123, 7 (1976) 199C.

[7] F. G. Fagan, V. R. W. Amarakoon, Am. Ceram. Soc. Bull. 72, 3 (1993) 119.

[8] Y. S. He, J. C. Campbell, R. C. Murphy, M. F. Arendt, J. S. Swinnea, J. Mater. Res. 8,12 (1993) 3131.

[9] S. A. Pianaro, P. R. Bueno, E. Longo, J. A. Varela, J. Mater. Sci. Lett. 14 (1995) 692.

[10] Z. R. Dai, Z. W. Pan, Z. L. Wang, Solid State Comm. 118 (2001) 351 .

[11] Z. R. Dai, J. L. Gole, J. D. Stout, Z. L. Wang, J. Phys. Chem. B 106, 6 (2002) 1274.

[12] K. Suito, N. Kawai, Y. Masuda, Mater. Res. Bull, 10 (1975) 677. [13] E. R. Leite, J. W. Gomes, M. M. Oliveira, E. J. H. Lee, E. Longo, J. A. Varela, C. A. Paskocimas, T. M. Boschi, F. Lanciotti, P. S. Pizani, P. C. Soares, J. Nanosci. Nanotech. 2, 2 (2002) 1. [14] G. V. Samsonov, The Oxide Handbook, $1^{\text {st }}$ Ed., Plenum Press, London (1973) p. 218.

[15] J. K. Jian, X. L. Chen, W. J. Wang, L. Dai, Y. P. Xu, Appl. Phys. A - Mater. 76, 2 (2003) 291.

(Rec. 04/09/03, Rev. 07/11/03, Ac. 05/12/03) 